K. MATSUSHITA KODAI MATH. J.

7 (1983), $61-72$

\title{
ON COMPACT RIEMANNIAN MANIFOLDS ADMITTING A CERTAIN FUNCTION
}

\author{
By KazUhiro Matsushita
}

\section{Introduction.}

Let $(M, g)$ be an $n$-dimensional complete Riemannian manifold. Then it is known that the existence of a function satisfying some differential equations on $(M, g)$ sometimes determines differential geometric properties of $(M, g)$. For example, if $(M, g)$ admits a non-trivial solution $f$ of the system of differential equations

$$
\nabla(d f)+k f g=0,
$$

for some positive constant $k$, then it is isometric to an $n$-dimensional Euclidean sphere $S^{n}(k)$ of radius $1 / \sqrt{k}$ (S. Ishihara and Y. Tashiro [5], M. Obata [7], [8]). In this paper, concerning with this result, we shall consider the problem: "Perturbing the above equality (1.1), can we restrict the topological structure of $(M, g)$ ?", and prove the following

THEOREM A. Let $(M, g)$ be an n-dimensional compact connected Riemannian manifold with the diameter $d(M)$ satisfying the inequality $\pi / 2<d(M)<3 \pi / 2$. Assume that $(M, g)$ admits a non-constant function $f$ satisfying following condition

$$
|\nabla(d f)(X, X)+f g(X, X)|<\varepsilon\left(d(M), f\left(P_{+}\right), f\left(P_{-}\right)\right)\|X\|^{2},
$$

for any $X(\neq 0) \in M_{x}(x \in M)$, where

$$
\begin{aligned}
0<\varepsilon & \left(d(M), f\left(P_{+}\right), f\left(P_{-}\right)\right) \\
& =\min \left\{\frac{f\left(P_{+}\right)}{4},-\frac{f\left(P_{-}\right)}{4},-\frac{f\left(P_{+}\right) \cos d(M)}{\cos d(M)+3}, \frac{f\left(P_{-}\right) \cos d(M)}{\cos d(M)+3}\right\},
\end{aligned}
$$

and $f\left(P_{+}\right)\left(f\left(P_{-}\right)\right.$, resp. $)=\max _{p \in M}\{f(P)\}\left(\min _{p \in M}\{f(P)\}\right.$, resp. $)$. Then $M$ is homeomorphic to an $n$-dimensional sphere.

Proposition B. Let $(M, g)$ be an n-dimensional compact connected Riemannian manifold and $H_{1}(M, g)$ the set of all the first eigen functions of the Laplace operator $\Delta$ of $(M, g)$. Assume that there exists a function $f \in H_{1}(M, g)$ satisfying

Received March 14, 1983 
the following condition

$$
|\nabla(d f)(X, X)+f g(X, X)|<\varepsilon\left(f\left(P_{+}\right), f\left(P_{-}\right)\right)\|X\|^{2}
$$

for all $X(\neq 0) \in M_{x}(x \in M)$, where

$$
0<\varepsilon\left(f\left(P_{+}\right), f\left(P_{-}\right)\right)<\min \left\{\frac{f\left(P_{+}\right)}{4},-\frac{f\left(P_{-}\right)}{4}\right\} .
$$

Then $M$ is homeomorphic an n-dimensional sphere.

The above problem has also been discussed by M. A. Pinsky [8] and S. Gallot [4]. The author wishes to express his deep gratitude to Prof. K. Sekigawa for useful comments and kind encouragement.

\section{Some estimations.}

In order to show our Theorem $\mathrm{A}$ in the next section, we have to prove several lemmas on the estimation. The proofs of the lemmas are essentially the same as those of M. A. Pinsky [8]. Let $(M, g)$ be an $n$-dimensional complete Riemannian manifold and assume that it admits a function $f$ satisfying $(*)$. Let $\gamma$ be a normal geodesic. We put

$$
\delta_{\gamma}(t)=\frac{d^{2}(f \circ \gamma)}{d t^{2}}(t)+(f \circ \gamma)(t) . \quad \text { along } \gamma .
$$

Then by the condition $(*)$ we get

$$
\left|\delta_{\gamma}(t)\right|<\varepsilon, \quad \text { along } \gamma \text {. }
$$

From (2.1), we get easily

$$
(f \circ \gamma)(t)=(f \circ \gamma)(0) \cos t+(f \circ \gamma)^{\prime}(0) \sin t+\int_{0}^{t} \delta_{\gamma}(u) \sin (t-u) d u .
$$

In particular, if $\gamma(0)$ is a critical point of $f,(2.3)$ reduces to

$$
(f \circ \gamma)(t)=(f \circ \gamma)(0) \cos t+\int_{0}^{t} \delta_{\gamma}(u) \sin (t-u) d u .
$$

Then taking account of (2.2) and (2.4), we get easily

$$
\begin{array}{ll}
\left\{f\left(P_{+}\right)+\varepsilon\right\} \cos t-\varepsilon \leqq(f \circ \gamma)(t) \leqq\left\{f\left(P_{+}\right)-\varepsilon\right\} \cos t+\varepsilon, & \text { for } 0 \leqq t \leqq \pi, \\
\left\{f\left(P_{-}\right)+\varepsilon\right\} \cos t-\varepsilon \leqq(f \circ \gamma)(t) \leqq\left\{f\left(P_{-}\right)-\varepsilon\right\} \cos t+\varepsilon, & \text { for } 0 \leqq t \leqq \pi, \\
\left\{f\left(P_{+}\right)-\varepsilon\right\} \cos t-3 \varepsilon<(f \circ \gamma)(t)<\left\{f\left(P_{+}\right)+\varepsilon\right\} \cos t+3 \varepsilon, & \text { for } \pi \leqq t \leqq 2 \pi, \\
\left\{f\left(P_{-}\right)-\varepsilon\right\} \cos t-3 \varepsilon<(f \circ \gamma)(t)<\left\{f\left(P_{-}\right)+\varepsilon\right\} \cos t+3 \varepsilon, & \text { for } \pi \leqq t \leqq 2 \pi .
\end{array}
$$

From (2.4), we get 


$$
\begin{aligned}
& (f \circ \gamma)^{\prime}(t)=-f\left(P_{+}\right) \sin t+\int_{0}^{t} \delta_{\gamma}(u) \cos (t-u) d u, \\
& (f \circ \gamma)^{\prime}(t)=-f\left(P_{-}\right) \sin t+\int_{0}^{t} \delta_{\gamma}(u) \cos (t-u) d u .
\end{aligned}
$$

Taking account of (2.2), (2.7) and $(2.7)^{\prime}$, we get

$$
\begin{array}{rrr}
(2.8) & -\left\{f\left(P_{+}\right)+\varepsilon\right\} \sin t \leqq(f \circ \gamma)^{\prime}(t) \leqq\left\{-f\left(P_{+}\right)+\varepsilon\right\} \sin t, & \text { for } 0 \leqq t \leqq \pi / 2, \\
(2.8)^{\prime} & -\left\{f\left(P_{-}\right)+\varepsilon\right\} \sin t \leqq(f \circ \gamma)^{\prime}(t) \leqq\left\{-f\left(P_{-}\right)+\varepsilon\right\} \sin t, & \text { for } 0 \leqq t \leqq \pi / 2, \\
(2.9) & \left\{-f\left(P_{+}\right)+\varepsilon\right\} \sin t-2 \varepsilon<(f \circ \gamma)^{\prime}(t)<-\left\{f\left(P_{+}\right)+\varepsilon\right\} \sin t+2 \varepsilon, \\
& \text { for } \pi / 2 \leqq t \leqq 3 \pi / 2, \\
(2.9)^{\prime} & \left\{-f\left(P_{-}\right)+\varepsilon\right\} \sin t-2 \varepsilon<(f \circ \gamma)^{\prime}(t)<\left\{-f\left(P_{-}\right)+\varepsilon\right\} \sin t+2 \varepsilon,
\end{array}
$$

We put

$$
\begin{aligned}
& t_{0}(\gamma)=\min \{t>0 ;(f \circ \gamma)(t)=-(f \circ \gamma)(0)\}, \\
& \bar{t}_{0}(\gamma)=\min \{t>0 ;(f \circ \gamma)(t)=(f \circ \gamma)(0)\}, \\
& t_{0}^{\prime}(\gamma)=\min \left\{t>0 ;(f \circ \gamma)^{\prime}(t)=0\right\} .
\end{aligned}
$$

Then we get the following

LEMMA 2.1. Let $\gamma$ be any normal geodesic starting from $P_{+}\left(P_{-}\right.$, resp.). Then we have

$$
\bar{t}_{0}(\gamma)>\pi, \quad t_{0}(\gamma)>\pi-\cos ^{-1} \frac{f\left(P_{+}\right)-\varepsilon}{f\left(P_{+}\right)+\varepsilon},
$$

and

$$
t_{0}^{\prime}(\gamma)>\pi-\sin ^{-1} \frac{2 \varepsilon}{f\left(P_{+}\right)+\varepsilon}>\pi-\cos ^{-1} \frac{f\left(P_{+}\right)-\varepsilon}{f\left(P_{+}\right)+\varepsilon}
$$

$$
\bar{t}_{0}(\gamma)>\pi, \quad t_{0}(\gamma)>\pi-\cos ^{-1} \frac{f\left(P_{-}\right)+\varepsilon}{f\left(P_{-}\right)-\varepsilon}
$$

and

$$
\left.t_{0}^{\prime}(\gamma)>\pi-\sin ^{-1} \frac{2 \varepsilon}{-f\left(P_{-}\right)+\varepsilon}>\pi-\cos ^{-1} \frac{f\left(P_{-}\right)+\varepsilon}{f\left(P_{-}\right)-\varepsilon}, \text { resp. }\right) \text {. }
$$

Proof. First, we show the first inequality of (2.10). By (2.5), we get

$$
\begin{array}{r}
\cos t-\frac{\varepsilon}{f\left(P_{+}\right)}(1-\cos t) \leqq \frac{(f \circ \gamma)(t)}{f\left(P_{+}\right)} \leqq \cos t+\frac{\varepsilon}{f\left(P_{+}\right)}(1-\cos t)<1, \\
\text { for } 0<t<\pi .
\end{array}
$$

Then, we see that $\bar{t}_{0}(\gamma)>\pi$. Next, we show the second inequality of (2.10). We have only to consider the case $0<t_{0}(\gamma)<\pi$. From (2.11), we get 


$$
\cos t_{0}(\gamma)-\frac{\varepsilon}{f\left(P_{+}\right)}\left(1-\cos t_{0}(\gamma)\right)<\frac{(f \circ \gamma)\left(t_{0}(\gamma)\right)}{f\left(P_{+}\right)}=-1 \text {, }
$$

and hence $t_{0}(\gamma)>\pi-\cos ^{-1} \frac{f\left(P_{+}\right)-\varepsilon}{f\left(P_{+}\right)+\varepsilon}$. Lastly, we show the third inequality of (2.10). We have only to consider the case $t_{0}^{\prime}(\gamma)<\pi$. If $t_{0}^{\prime}(\gamma) \leqq \pi / 2$, then from (2.8), we get $f\left(P_{+}\right)<\varepsilon$, which contradicts to $f\left(P_{+}\right)>\varepsilon>0$. Thus, we may assume that $t_{0}^{\prime}(\gamma)>\pi / 2$. From (2.9), we get

and hence

$$
f\left(P_{+}\right) \sin t_{0}^{\prime}(\gamma)<\varepsilon\left(2-\sin t_{0}^{\prime}(\gamma)\right),
$$

$$
t_{0}^{\prime}(\gamma)>\pi-\sin ^{-1} \frac{2 \varepsilon}{f\left(P_{+}\right)+\varepsilon} .
$$

Since $f\left(P_{+}\right)>\varepsilon>0$, we get

$$
\frac{2 \varepsilon}{f\left(P_{+}\right)+\varepsilon}<\frac{2 \sqrt{\varepsilon f\left(P_{+}\right)}}{f\left(P_{+}\right)+\varepsilon} .
$$

Thus, from (2.12) and (2.13), we get

$$
\begin{aligned}
t_{0}^{\prime}(\gamma) & >\pi-\sin ^{-1} \frac{2 \sqrt{\varepsilon f\left(P_{+}\right)}}{f\left(P_{+}\right)+\varepsilon} \\
& =\pi-\cos ^{-1} \frac{f\left(P_{+}\right)-\varepsilon}{f\left(P_{+}\right)+\varepsilon} .
\end{aligned}
$$

Similarly, we may also show (2.10)'.

Q.E.D.



Fig. I 




Fig. II

From (2.5), (2.6), (2.8) (2.10), we have the figures I, II. Moreover, from (2.5)', $(2.6)^{\prime},(2.8)^{\prime} \sim(2.10)^{\prime}$, we have also the similar figures. However, we omit to write them.

LEMMA 2.2. Let $\gamma$ be any normal geodesic starting from $P_{+}\left(P_{-}\right.$, resp. $)$, and $\gamma\left(t_{c}\right)$ the conjugate point of $P_{+}=\gamma(0)\left(P_{-}=\gamma(0)\right.$, resp. $)$ along $\gamma$. Then we have

$$
t_{c}>\pi-\sin ^{-1} \frac{2 \varepsilon}{f\left(P_{+}\right)+\varepsilon},
$$

$$
\left.t_{c}>\pi-\sin ^{-1} \frac{2 \varepsilon}{-f\left(P_{-}\right)+\varepsilon}, \text { resp. }\right) \text {. }
$$

Proof. Let $Y$ be a Jacobi field along $\gamma$ such that $Y(0)=0,\langle Y, \dot{\gamma}\rangle=0$ and $Y\left(t_{c}\right)=0$. First, we assume that $\left\|Y\left(t_{0}\right)\right\|=1$ for some $t_{0}\left(0<t_{0}<t_{c}\right)$. Let $\sigma_{t_{0}}(s)$ be the normal geodesic starting from $\gamma\left(t_{0}\right)$ such that $\dot{\sigma}_{t_{0}}(0)=Y\left(t_{0}\right)$, and let $\gamma_{s}(t)$ be the normal geodesic from $P_{+}$to $\sigma_{t_{0}}(s)$ such that $\alpha(t, s)=\gamma_{s}(t)$ is a smooth variation of $\gamma$. Then we may easily see that the associated variational field $Z(t)=$ $d \alpha\left(\left.\frac{\partial}{\partial s}\right|_{(t, 0)}\right)$ along $\gamma$ coincides with the given Jacobi field $Y$. By $L_{s}$ we denote the length of the geodesic $\gamma_{s}$. First of all, we get

$$
\left.\frac{d L_{s}}{d s}\right|_{s=0}=0 \text {. }
$$

In view of the second variational formula, we get 


$$
\left.\frac{d^{2} L_{s}}{d s^{2}}\right|_{s=0}=\left\langle Y^{\prime}\left(t_{0}\right), Y\left(t_{0}\right)\right\rangle
$$

where $Y^{\prime}=\nabla_{j} Y$. From (2.4), we get

$$
f\left(\sigma_{t_{0}}(s)\right)=f\left(\gamma_{s}\left(L_{s}\right)\right)=(f \circ \gamma)(0) \cos L_{s}+\int_{0}^{L_{s}} \delta_{\gamma_{s}}(u) \sin \left(L_{s}-u\right) d u .
$$

From (2.17), taking account of (2.7) and (2.15), we get

$$
\begin{aligned}
& \left.\frac{d^{2} L_{s}}{d s^{2}}\right|_{s=0}(f \circ \gamma)^{\prime}\left(t_{0}\right)+\int_{0}^{t_{0}}\left(\left.\frac{d^{2} \delta_{\gamma_{s}}(u)}{d s^{2}}\right|_{s=0}\right) \sin \left(t_{0}-u\right) d u \\
& =\delta_{\sigma_{t_{0}}}(0)-\delta_{\gamma}\left(t_{0}\right)+(f \circ \gamma)^{\prime \prime}\left(t_{0}\right) .
\end{aligned}
$$

We now assume that $(f \circ \gamma)^{\prime}\left(t_{0}\right) \neq 0$. Then, from (2.18), we get

$$
\left.\frac{d^{2} L_{s}}{d s^{2}}\right|_{s=0}=\frac{F\left(t_{0}\right)}{(f \circ \gamma)^{\prime}\left(t_{0}\right)}
$$

where

$$
F(t)=(f \circ \gamma)^{\prime \prime}(t)-\delta_{\gamma}(t)+\delta_{\sigma_{t}}(0)-\int_{0}^{t}\left(\left.\frac{d^{2} \delta_{\gamma_{s}}(u)}{d s^{2}}\right|_{s=0}\right) \sin (t-u) d u
$$

Next, we assume that $\left\|Y\left(t_{0}\right)\right\| \neq 1$. We put $\tilde{Y}(t)=\frac{1}{\left\|Y\left(t_{0}\right)\right\|} Y(t)$. Then we get

$$
\left\langle\tilde{Y}^{\prime}\left(t_{0}\right), \tilde{Y}\left(t_{0}\right)\right\rangle=\frac{\left\langle Y^{\prime}\left(t_{0}\right), Y\left(t_{0}\right)\right\rangle}{\left\langle Y\left(t_{0}\right), Y\left(t_{0}\right)\right\rangle} .
$$

Therefore, applying the formulas (2.16), (2.19) and (2.20), we have

$$
\frac{\left\langle Y^{\prime}(t), Y(t)\right\rangle}{\langle Y(t), Y(t)\rangle}=\frac{F(t)}{(f \circ \gamma)^{\prime}(t)} \text {. }
$$

The rest of the proof is similar to the one of Lemma 5 in [8]. Similarly, we may also show $(2.14)^{\prime}$.

Q.E.D.

By $C\left(P_{+}\right)\left(C\left(P_{-}\right)\right.$, resp. $)$we denote the cut locus of $P_{+}\left(P_{-}\right.$, resp. $)$. Then we have the following

LEMMA 2.3. Let $d\left(P_{+}, C\left(P_{+}\right)\right)\left(d\left(P_{-}, C\left(P_{-}\right)\right)\right.$, resp. $)$be the distance from $P_{+}$ $\left(P_{-}\right.$, resp. $)$to the cut locus $C\left(P_{+}\right)\left(C\left(P_{-}\right)\right.$, resp. $)$. Then

$$
\begin{aligned}
& d\left(P_{+}, C\left(P_{+}\right)\right)>\pi-\sin ^{-1} \frac{2 \varepsilon}{f\left(P_{+}\right)+\varepsilon}, \\
& \left.d\left(P_{-}, C\left(P_{-}\right)\right)>\pi-\sin ^{-1} \frac{2 \varepsilon}{-f\left(P_{-}\right)+\varepsilon}, \text { resp. }\right) .
\end{aligned}
$$

The proof of this Lemma is similar to the one of Lemma 6 in [8]. So, we omit it. Furthermore, we have the following 
Lemma 2.4. Let $d(M)$ be the diameter of $(M, g)$. Then we have

$$
\pi-\sin ^{-1} \frac{2 \varepsilon}{f\left(P_{+}\right)+\varepsilon}<d\left(P_{+}, P_{-}\right) \leqq d(M)<2 \pi-\cos ^{-1} \frac{-3 \varepsilon}{f\left(P_{+}\right)+\varepsilon}
$$

and

$$
\pi-\sin ^{-1} \frac{2 \varepsilon}{-f\left(P_{-}\right)+\varepsilon}<d\left(P_{+}, P_{-}\right) \leqq d(M)<2 \pi-\cos ^{-1} \frac{3 \varepsilon}{f\left(P_{-}\right)-\varepsilon} .
$$

Proof. The first inequality of (2.23) follows immediately from (2.10), and the second one of $(2.23)$ is trivial. If $d(M) \leqq \pi$, the third inequality is trivial because of $\pi \leqq 2 \pi-\cos ^{-1} \frac{-3 \varepsilon}{f\left(P_{+}\right)+\varepsilon}$ (cf. Fig. I). So we assume that $d(M)>\pi$. From the condition $\left(^{*}\right)$ in Theorem A, it follows that

and hence

$$
\varepsilon<\frac{-f\left(P_{+}\right) \cos d(M)}{\cos d(M)+3},
$$

$$
-\frac{3 \varepsilon}{f\left(P_{+}\right)+\varepsilon}>\cos d(M)=\cos (2 \pi-d(M))
$$

Thus, we have finally $\cos ^{-1} \frac{-3 \varepsilon}{f\left(P_{+}\right)+\varepsilon}<2 \pi-d(M)$. Similarly, we may also show (2.24).

Q.E.D.

LEMMA 2.5. We have the following inequalities:

$$
\begin{aligned}
& \cos ^{-1} \frac{-\varepsilon}{f\left(P_{+}\right)-\varepsilon}<\pi-\cos ^{-1} \frac{f\left(P_{+}\right)-\varepsilon}{f\left(P_{+}\right)+\varepsilon}\left(<\pi-\sin ^{-1} \frac{2 \varepsilon}{f\left(P_{+}\right)-\varepsilon}\right), \\
& \cos ^{-1} \frac{-\varepsilon}{f\left(P_{+}\right)-\varepsilon}<\pi-\sin ^{-1} \frac{2 \varepsilon}{-f\left(P_{-}\right)+\varepsilon}, \\
& \cos ^{-1} \frac{\varepsilon}{f\left(P_{-}\right)+\varepsilon}<\pi-\cos ^{-1} \frac{f\left(P_{-}\right)+\varepsilon}{f\left(P_{-}\right)-\varepsilon}\left(<\pi-\sin ^{-1} \frac{2 \varepsilon}{-f\left(P_{-}\right)-\varepsilon}\right), \\
& \cos ^{-1} \frac{\varepsilon}{f\left(P_{-}\right)+\varepsilon}<\pi-\sin ^{-1} \frac{2 \varepsilon}{f\left(P_{+}\right)+\varepsilon} .
\end{aligned}
$$

Proof. First, we show the first inequality of (2.25). Let us define a function $g(t)$ by

$$
g(t)=\left\{f\left(P_{+}\right)-\varepsilon\right\} \cos t+\varepsilon \quad(0 \leqq t \leqq \pi),
$$

which is easily found to be a monotonously decreasing function satisfying

$$
g\left(\cos ^{-1} \frac{-\varepsilon}{f\left(P_{+}\right)-\varepsilon}\right)=0, \quad g(\pi)=-f\left(P_{+}\right) \div 2 \varepsilon<0 .
$$

By the definition of $g(t)$ and the condition (*), we get immediately 


$$
\begin{aligned}
g\left(\pi-\cos ^{-1} \frac{f\left(P_{+}\right)-\varepsilon}{f\left(P_{+}\right)+\varepsilon}\right) & =\left\{f\left(P_{+}\right)-\varepsilon\right\} \cos \left(\pi-\cos ^{-1} \frac{f\left(P_{+}\right)-\varepsilon}{f\left(P_{+}\right)+\varepsilon}\right)+\varepsilon \\
& =-\frac{\left\{f\left(P_{+}\right)-\varepsilon\right\}^{2}}{\left\{f\left(P_{+}\right)+\varepsilon\right\}}+\varepsilon>0,
\end{aligned}
$$

and hence

$$
\cos ^{-1} \frac{-\varepsilon}{f\left(P_{+}\right)-\varepsilon}<\pi-\cos ^{-1} \frac{f\left(P_{+}\right)-\varepsilon}{f\left(P_{+}\right)+\varepsilon} .
$$

Thus, we have the first inequality of (2.25). Next, we show the second inequality of (2.25). Taking account of Fig. I, we get

$$
f\left(P_{+}\right)+2 \varepsilon>-f\left(P_{-}\right)>f\left(P_{+}\right)-2 \varepsilon .
$$

From (2.28), we get

$$
\pi-\sin ^{-1} \frac{2 \varepsilon}{f\left(P_{+}\right)+3 \varepsilon}>\pi-\sin ^{-1} \frac{2 \varepsilon}{-f\left(P_{-}\right)+\varepsilon}>\pi-\sin ^{-1} \frac{2 \varepsilon}{f\left(P_{+}\right)-\varepsilon} .
$$

On the other hand, by the condition $(*)$, we get

$$
\frac{\sqrt{\left\{f\left(P_{+}\right)-3 \varepsilon\right\}\left\{f\left(P_{+}\right)+\varepsilon\right\}}}{f\left(P_{+}\right)-\varepsilon}>\frac{\varepsilon}{f\left(P_{+}\right)-\varepsilon} \quad(>0) .
$$

From (2.30), we get

$$
\begin{aligned}
& \cos \left(\pi-\cos ^{-1} \frac{\sqrt{\left\{f\left(P_{+}\right)-3 \varepsilon\right\}\left\{f\left(P_{+}\right)+\varepsilon\right\}}}{f\left(P_{+}\right)-\varepsilon}\right) \\
& =-\frac{\sqrt{\left\{f\left(P_{+}\right)-3 \varepsilon\right\}\left\{f\left(P_{+}\right)+\varepsilon\right\}}}{f\left(P_{+}\right)-\varepsilon}<-\frac{\varepsilon}{f\left(P_{+}\right)-\varepsilon} \\
& =\cos \left(\cos ^{-1} \frac{-\varepsilon}{f\left(P_{+}\right)-\varepsilon}\right),
\end{aligned}
$$

and hence

$$
\pi-\cos ^{-1}\left(\frac{\sqrt{\left\{f\left(P_{+}\right)-3 \varepsilon\right\}\left\{f\left(P_{+}\right)+\varepsilon\right\}}}{f\left(P_{+}\right)-\varepsilon}\right)>\cos ^{-1} \frac{-\varepsilon}{f\left(P_{+}\right)-\varepsilon} .
$$

Finally the formula (2.31) together with

$$
1-\left(\frac{2 \varepsilon}{f\left(P_{+}\right)-\varepsilon}\right)^{2}=\left\{\frac{\sqrt{\left\{f\left(P_{+}\right)-3 \varepsilon\right\}\left\{f\left(P_{+}\right)+\varepsilon\right\}}}{f\left(P_{+}\right)-\varepsilon}\right\}^{2},
$$

implies

$$
\pi-\sin ^{-1} \frac{2 \varepsilon}{f\left(P_{+}\right)-\varepsilon}=\pi-\cos ^{-1} \frac{\sqrt{\left\{f\left(P_{+}\right)-3 \varepsilon\right\}\left\{f\left(P_{+}\right)+\varepsilon\right\}}}{f\left(P_{+}\right)-\varepsilon}>\cos ^{-1} \frac{-\varepsilon}{f\left(P_{+}\right)-\varepsilon} .
$$

Similarly, we may also show (2.26).

Q.E.D. 


\section{Proof of Theorem A.}

In this section, we give a proof of Theorem $\mathrm{A}$ based on the estimations obtained in $\S 2$.

LEMma 3.1. There is a positive number $r$ such that $M=B_{r}\left(P_{+}\right) \cup B_{r}\left(P_{-}\right)$, and

$$
\begin{aligned}
& \cos ^{-1} \frac{-\varepsilon}{f\left(P_{+}\right)-\varepsilon} \leqq r \\
& \quad \leqq \min \left\{\pi-\sin ^{-1} \frac{2 \varepsilon}{f\left(P_{+}\right)+\varepsilon}, \pi-\sin ^{-1} \frac{2 \varepsilon}{-f\left(P_{-}\right)+\varepsilon}\right\}, \\
& \cos ^{-1} \frac{\varepsilon}{f\left(P_{-}\right)+\varepsilon} \leqq r \\
& \quad \leqq \min \left\{\pi-\sin ^{-1} \frac{2 \varepsilon}{-f\left(P_{-}\right)+\varepsilon}, \pi-\sin ^{-1} \frac{2 \varepsilon}{f\left(P_{+}\right)+\varepsilon}\right\},
\end{aligned}
$$

where $B_{r}\left(P_{+}\right)\left(B_{r}\left(P_{-}\right)\right.$, resp. $)$denotes the open ball of the center $P_{+}\left(P_{-}\right.$, resp. $)$and of radius $r$.

Proof. From Lemmas 2.3 and 2.5, we may easily see that there exists a positive number $r$ satisfying the condition (3.1). Let $\gamma$ ( $\sigma$, resp.) be any normal geodesic starting from $P_{+}\left(P_{-}\right.$, resp.). From Lemma 2.4, taking account of Fig. I, we get

$$
\begin{array}{r}
(f \circ \gamma)(t)<0 \quad \text { for } \quad \cos ^{-1} \frac{-\varepsilon}{f\left(P_{+}\right)-\varepsilon} \leqq t \leqq d(M) \\
<2 \pi-\cos ^{-1} \frac{-3 \varepsilon}{f\left(P_{+}\right)+\varepsilon} .
\end{array}
$$

Similarly, we get

$$
\begin{array}{r}
(f \circ \sigma)(s)>0 \text { for } \cos ^{-1} \frac{\varepsilon}{f\left(P_{-}\right)+\varepsilon} \leqq s \leqq d(M) \\
<2 \pi-\cos ^{-1} \frac{3 \varepsilon}{f\left(P_{-}\right)-\varepsilon} .
\end{array}
$$

We suppose that $M-\left(B_{r}\left(P_{+}\right) \cup B_{r}\left(P_{-}\right)\right) \neq \varnothing$. Let $x \in M-\left(B_{r}\left(P_{+}\right) \cup B_{r}\left(P_{-}\right)\right)$and $\gamma(\sigma$, resp. $)$ be the minimal geodesic from $P_{+}\left(P_{-}\right.$, resp. $)$to $x=\gamma\left(t_{0}\right)\left(x=\sigma\left(s_{0}\right)\right.$, resp.). Then, by the choice of $x$, we see that

and

$$
r \leqq t_{0}<2 \pi-\cos ^{-1} \frac{-3 \varepsilon}{f\left(P_{+}\right)+\varepsilon}
$$

Thus, we have

$$
r \leqq s_{0}<2 \pi-\cos ^{-1} \frac{3 \varepsilon}{f\left(P_{-}\right)-\varepsilon}
$$




$$
f(x)=(f \circ \gamma)\left(t_{0}\right)<0 \text { and } f(x)=(f \circ \sigma)\left(s_{0}\right)>0,
$$

which is a contradiction.

Q.E.D.

LEMMA 3.2. Suppose that $r$ is a positive number satisfying the condition in Lemma 3.1, and $\gamma$ any normal geodesic starting from $P_{+}$. Then there is a unique point $x$ on $\gamma$ such that $d\left(P_{+}, x\right)=d\left(P_{-}, x\right)<r$.

Proof. We put

$$
h(t)=d\left(P_{+}, r(t)\right)-d\left(P_{-}, \gamma(t)\right) \quad \text { for } \quad 0 \leqq t \leqq \pi-\sin ^{-1} \frac{2 \varepsilon}{f\left(P_{+}\right)+\varepsilon} .
$$

Then, by Lemma 3.1 , we get

$$
\begin{aligned}
h(0)=-d\left(P_{-}, P_{+}\right)<0, & \\
h\left(\pi-\sin ^{-1} \frac{2 \varepsilon}{f\left(P_{+}\right)+\varepsilon}\right)=\pi & -\sin ^{-1} \frac{2 \varepsilon}{f\left(P_{+}\right)+\varepsilon} \\
& \quad-d\left(P_{-}, \gamma\left(\pi-\sin ^{-1} \frac{2 \varepsilon}{f\left(P_{+}\right)+\varepsilon}\right)\right)>0 .
\end{aligned}
$$

Thus, by the intermediate value theorem, there is a point $x$ such that $d\left(P_{+}, x\right)$ $=d\left(P_{-}, x\right)$. First, we assume that $d\left(P_{+}, x\right)>r$. Then, by Lemma 3.1, we see that $d\left(P_{-}, x\right)<r$, which contradicts to $d\left(P_{+}, x\right)=d\left(P_{-}, x\right)$. Thus, $d\left(P_{+}, x\right)<r$. Next, we assume that there exist $x_{1}, x_{2}$ on $\gamma\left(x_{1} \neq x_{2}\right)$ such that $d\left(P_{+}, x_{i}\right)=$ $d\left(P_{-}, x_{i}\right), i=1,2$. Moreover, we may assume that $d\left(P_{+}, x_{1}\right)<d\left(P_{+}, x_{2}\right)$. Then, we get

$$
\begin{aligned}
d\left(P_{-}, x_{2}\right)=d\left(P_{+}, x_{2}\right) & =d\left(P_{+}, x_{1}\right)+d\left(x_{1}, x_{2}\right) \\
& =d\left(P_{-}, x_{1}\right)+d\left(x_{1}, x_{2}\right) .
\end{aligned}
$$

Let $\sigma$ be the minimal geodesic from $x_{1}$ to $P_{-}$. Since $r<\pi-\sin ^{-1} \frac{2 \varepsilon}{f\left(P_{+}\right)+\varepsilon}$, there exists a unique minimal geodesic from $x_{1}$ to $x_{2}$. Furthermore, by (3.2), we see that $\sigma \cup\left(-\left.\gamma\right|_{\left[x_{1}, x_{2}\right]}\right)$ is a minimal geodesic from $x_{2}$ to $P_{-}$, and hence $\sigma \cup\left(-\left.\gamma\right|_{\left.L_{1}, x_{2}\right]}\right)$ $=-\gamma$. Therefore, it follows that $P_{+}=P_{-}$. But, this is a contradiction.

Q.E.D.

Now, we are in a position to prove Theorem A. Let $S^{n}$ be an $n$-dimensional unit sphere, and $(p, q)\left(p, q \in S^{n}\right)$ be an antipodal pair. Let $I: S_{p}^{n} \rightarrow M_{P_{+}}$be a linear isometry. By Lemma 3.2, there is a real number $t_{0}$ such that $d\left(P_{+}, \exp _{P_{+}}\left(t_{0} v\right)\right)=d\left(P_{-}, \exp _{P_{+}}\left(t_{0} v\right)\right)$. Then we put $k(v)=t_{0} v \in M_{P_{+}}-\{0\}$. By means of $k(v)$, we may define a map $\phi: S^{n} \rightarrow M$ by 
ON COMPACT RIEMANNIAN MANIFOLDS

$$
\phi(x)= \begin{cases}P_{+}, & \text {for } x=p, \\ \exp _{P_{+}}\left(\frac{2 d(x, p)}{\pi}\left(k \circ I \circ \exp _{p}^{-1}(x)\right)\right), & \text { for } x \in \overline{B_{\pi / 2}(p)}-\{p\}, \\ \exp _{P_{-}}\left(\frac{2 \pi(x, q)}{\pi}\left(\exp _{P_{-}}^{-1} \circ \exp _{P_{+}} \circ k \circ I \circ \exp _{p}^{-1}(x)\right)\right), & \text { for } x \in \overline{B_{\pi / 2}(q)}-\{q\}, \\ P_{-}, & \text {for } x=q,\end{cases}
$$

where $\tilde{d}$ denotes the distance on $S^{n}$.

By the similar arguments as those of [2] (Theorem 6.1), we may show that $\psi$ is continuous, injective and surjective, and hence $\phi$ is a homeomorphism from $S^{n}$ to $M$ by the compactness of $S^{n}$ and $M$. This completes the proof of Theorem A.

Lastly, we give an outline of the proof of Proposition B. Let $f$ be a first eigen function of the Laplace operator $\Delta$ of $(M, g)$ satisfying the condition $\left.{ }^{* *}\right)$ in Proposition B. Then, by Courant's nordal domain theorem ([3]), we see that the number of the connected components of $M-f^{-1}(0)$ is 2. From Lemma 3.1, we see that $f$ is negative (positive, resp.) on $\left(B_{\alpha}\left(P_{+}\right)\right)^{c}, \alpha=\cos ^{-1} \frac{-\varepsilon}{f\left(P_{+}\right)-\varepsilon}$ $\left(\left(B_{\beta}\left(P_{-}\right)\right)^{c}, \beta=\cos ^{-1} \frac{\varepsilon}{f\left(P_{-}\right)+\varepsilon}\right.$, resp. $)$, and hence $M=B_{r}\left(P_{-}\right) \cup B_{r}\left(P_{+}\right)$. The rest of the proof is similar as above.

Remark. Here we give a remark on the sign of $f\left(P_{-}\right)$in the Theorem A. By (2.5) for $t=\pi$ we get $(f \circ \gamma)(\pi)<-f\left(P_{+}\right)+2 \varepsilon$. Therefore, $f\left(P_{+}\right)>0$ and $\varepsilon<f\left(P_{+}\right) / 2$ imply $f\left(P_{-}\right)<0$.

\section{REFERENCES}

[1] M. Berger, P. Gauduchon and E. Mazert, Le Spectre d'une Variété Riemannienne, Lecture Note in Math., Vol. 194, Springer-Verlag, Berlin, 1971.

[2] J. Cheeger and D. G. Ebin, Comparison theorems in Riemannian geometry, North Holland, Amsterdam, 1975.

[3] S. Y. Cheng, Eigenfunctions and Nodal sets, Comment. Math. Helvetici, 51 (1976), 43-55.

[4] G. Gallot, Un theoreme de pincement et une estimation sur la premiere valeur proper du laplacien dune variété riemannienne, C. R. Acad. Sci. Paris, 289 (1979), $441-444$.

[5] S. IshiHARA AND Y. TASHIRo, On Riemannian manifolds admitting a concircular transformation, Math. J. Okayama Univ., 9 (1959), 19-47.

[6] М. Овата, Certain conditions for a Riemannian manifold to be isometric with a sphere, J. Math. Soc. Japan, 14 (1962), 333-340.

[7] M. OBATA, Riemannian manifolds admitting a solution of a certain system of differential equations, Proc. United States-Japan Sem. Differential Geometry, Kyoto, 1965, Nippon Hyoronsha, Tokyo (1966), 101-114. 
[8] M. A. Pinsky, A Topological Version of Obata's Sphere Theorem, J. Diff. Geom., 14 (1979), 369-376.

Department of Mathematics

Tokyo Institute of Technology 